\title{
Catalytic living ring-opening metathesis polymerization with Grubbs' second- and third-generation catalysts
}

\author{
Mohammad Yasiri,2, Peng Liu' ${ }^{1,2}$, Iris K. Tennie' and Andreas F. M. Kilbinger ${ }^{1}{ }^{1 \star}$ \\ In a conventional living ring-opening metathesis polymerization (ROMP), an equal number of ruthenium complexes to the num- \\ ber of polymer chains synthesized are required. This can lead to high loadings of ruthenium complexes when aiming for shorter \\ polymers. Here, a reversible chain-transfer agent was used to produce living ROMP polymers from norbornene derivatives \\ using catalytic amounts of Grubbs' ruthenium complexes. The polymers obtained by this method showed all of the character- \\ istics of a living polymerization (that is, good molecular weight control, narrow molecular weight dispersities and the ability to \\ form block copolymers). Monomers carrying functional moieties such as ferrocene, coumarin or a triisopropylsilyl-protected \\ primary alcohol could also be catalytically polymerized in a living fashion. The method presented follows a degenerative chain- \\ transfer process and is more economical and environmentally friendly compared with previous living ROMP procedures as it \\ utilizes only catalytic amounts of costly and toxic ruthenium complexes.
}

R ing-opening metathesis polymerization (ROMP) is a polymerization driven by the relief of the ring strain of cyclic olefins to form polymers ${ }^{1}$. Monomers such as norbornene derivatives can be polymerized in a living fashion due to the slow rates of irreversible chain-transfer events and termination reactions ${ }^{2}$ compared with the relatively fast propagation rates. Typical metathesis initiators for ROMP are based on ruthenium ${ }^{3}$ or molybdenum ${ }^{4,5}$. The high tolerance of ruthenium complexes towards many polar functional groups, water and oxygen has made them popular choices in organic as well as polymer chemistry ${ }^{6}$. The first and third generations of Grubbs' metathesis catalysts (G1 and G3, respectively; Fig. 1) are most commonly used for polymer synthesis due to their high initiation-to-propagation-rate ratio $^{7,8}$. Thus, ROMP has emerged as one of the most popular polymerization techniques due to its high functional group tolerance and the possibility of obtaining narrow dispersity polymers under mild reaction conditions and short reaction times.

Functional ROMP polymers carrying ferrocenes ${ }^{9}$, chromophores $^{10}$, fluorophores ${ }^{11}$ or reactive functional groups $s^{12-14}$ attached to the monomer units have been synthesized. ROMP polymers find applications as electroactive ${ }^{15}$, mechanoresponsive $e^{16,17}$ or watersoluble polymers ${ }^{18}$. Recently, tandem ring-opening/ring-closing polymerizations of cycloalkenes attached to other cycloalkenes ${ }^{19}$ or a terminal alkyne ${ }^{20-22}$ have been reported. Over the past decade, a variety of methods for the preparation of mono and bis-end-functional (telechelic) ROMP polymers have been developed ${ }^{23-27}$. ROMP polymers have also been prepared for biological/medicinal applications $^{28-30}$, electronics ${ }^{31-33}$, battery separators ${ }^{34}$, anion exchange membranes $^{35}$ and many others.

In a conventional living ROMP, the ruthenium carbene complex represents the propagating species located at the end of every polymer chain. For this reason, equimolar amounts of ruthenium complex with respect to the polymer chains are required. This leads to rather high ruthenium complex loadings if short polymer chains are aimed for. The associated increased costs and high levels of ruthenium contamination can make the synthesis of short polymer chains via a conventional living ROMP unattractive.

Catalytic methods for the preparation of shorter ROMP polymers have been reported. However, these methods rely on irreversible chain-transfer agents (CTAs). The polymerizations are therefore non-living, showing broad molecular weight dispersities, meaning that the transfer agents cannot be used to prepare block copolymers ${ }^{36,37}$. One way of achieving control over molecular weights and molecular weight dispersities in polymerizations is degenerative chain transfer. Here, a CTA is used to reversibly terminate the chain growth of polymers. This concept has been successfully employed in controlled radical polymerizations (via the reversible addition-fragmentation chain-transfer (RAFT) process) ${ }^{38}$, degenerative chain growth of polyolefins $^{39-41}$, ring-opening polymerizations of cyclic esters ${ }^{42}$ and cationic RAFT polymerization ${ }^{43}$.

Recently, our group has reported a method based on a degenerative reversible chain-transfer mechanism ${ }^{44}$ that used only catalytic amounts of ruthenium complex (G3) for living ROMP. However, we were not able to reproduce the data shown in that report and therefore retracted the article ${ }^{45}$. Nonetheless, the degenerative chain-transfer mechanism reported in the retracted paper could be reproduced and confirmed, but the CTA (CTA1; Fig. 1) gives much broader molecular weight dispersities and poorer molecular weight control than was reported in the retracted paper. Here, we report two different CTAs (CTA2 and CTA3; Fig. 1) that follow the originally proposed mechanism, and give kinetic evidence as to why these work better than CTA1.

Therefore, we report here a catalytic living ROMP with a reversible CTA (CTA2 or CTA3) that exploits a degenerative reversible chain-transfer polymerization mechanism requiring only catalytic amounts of the ruthenium-based Grubbs' second- (G2; Fig. 1) and third-generation (G3) metathesis catalysts, yielding polymers with good molecular weight control and narrow molecular weight dispersities. 


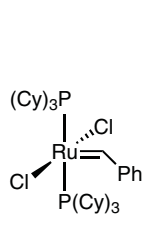

G1

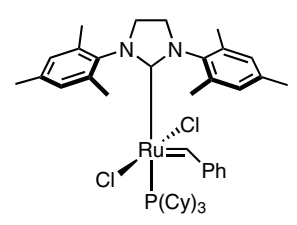

G2

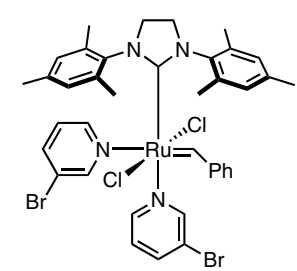

G3

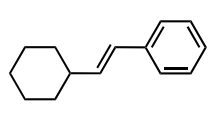

CTAO

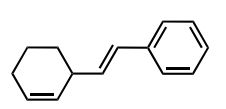

CTA1

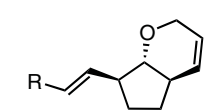

CTA2 $\mathrm{R}=$ phenyl $(\mathrm{Ph})$ CTA3 $\mathrm{R}=$ cyclohexyl (Cy)

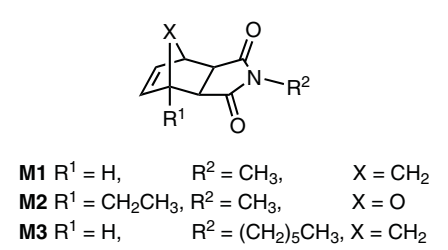

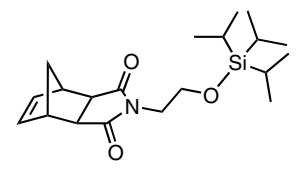

M4

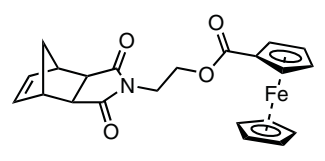

M5

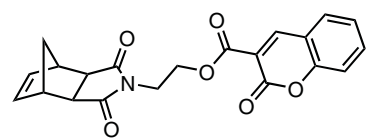

M6

M3 $\mathrm{R}^{1}=\mathrm{H}, \quad \mathrm{R}^{2}=\left(\mathrm{CH}_{2}\right)_{5} \mathrm{CH}_{3}, \mathrm{X}=\mathrm{CH}_{2}$

Fig. 1 Structure of the metathesis catalysts, CTAs and monomers investigated in this study. The metathesis catalysts include Grubbs' first- (G1), second- (G2) and third-generation (G3) ruthenium carbene complexes. The CTAs include (E)-(2-cyclohexylvinyl) benzene (CTA0), (E)-(2-(cyclohex-2-en-1-yl)vinyl)benzene (CTA1), (E)-7-styryl-2,4a,5,6,7,7a-hexahydrocyclopenta[b]pyran (CTA2) and (E)-7-(2-cyclohexylvinyl)2,4a,5,6,7,7a-hexahydrocyclopenta[b]pyran (CTA3). The monomers include exo- $N$-methyl-norbornenecarboximide (M1), exo-4-ethyl- $N$-methyl-7oxanorbornenecarboximide (M2), exo- $N$-hexyl-norbornenecarboximide (M3), exo- $N$-triisopropylsilyloxyethyl-norbornenecarboximide (M4), exo-N-ferrocenylcarbonyloxy-ethyl-norbornenecarboximide (M5) and exo- $\mathrm{N}$-coumarin-3-carbonyloxy-ethyl-norbornenecarboximide (M6).

\section{Results and discussion}

Our group recently published a report on functional metathesis catalysts showing that a structural analogue of CTA2 reacted with a propagating ruthenium carbene to transfer a cyclohexenyl end group onto the polymer chain ends while regenerating the ruthenium benzylidene complex ${ }^{46}$. This prompted us to exploit CTA2 in catalytic living ROMP. We believed that CTA2, similar to its structural analogue, could be easily prepared from a functional norbornene derivative in a tandem ring-opening/ring-closing reaction sequence.

We observed that CTA2 reacted rapidly with G3-initiated propagating ROMP polymers to give end-functional polymers carrying the bicyclic fragment of CTA2 at the chain end. The exocyclic double bond of CTA2 is sterically hindered, similar to the main chain double bonds of poly(norbornene) derivatives. Due to the branching in both allylic positions, these double bonds do not readily undergo secondary metathesis reactions. We therefore concluded that CTA2 reacted first with the propagating ruthenium carbene via its endocyclic double bond in a ring-opening/ringclosing sequence.

To prove the unreactive nature of the exocyclic double bond of CTA2, we synthesized the model compound CTA0 whose exocyclic double bond resembles that of CTA1 and CTA2 but lacks the endocyclic double bond. When CTA0 (10 equiv.) was added to a G3-initiated exo-N-methyl-norbornenecarboximide (M1) solution (POLY-G3, prepared by adding 20 equiv. M1 to a solution of G3 in $\mathrm{CD}_{2} \mathrm{Cl}_{2}$; see Supplementary Information), no reaction could be observed by ${ }^{1} \mathrm{H}$ NMR spectroscopy after $10 \mathrm{~min}$ at room temperature. Therefore, we concluded that CTA2 must react with its endocyclic double bond first, followed by a ring-closing reaction with the exocyclic double bond. This shows that the exocyclic double bond of CTA2 is reactive towards an intramolecular ring-closing metathesis reaction but not reactive in a bimolecular metathesis reaction step with a propagating ruthenium carbene complex.

When comparing CTA1 and CTA2, it was not immediately apparent to us which of the two would react faster with a POLY-G3 carbene complex. Both reagents carry a strainless, six-membered unsaturated ring, and the exocyclic double bond is virtually identical in both cases. We therefore prepared two POLY-G3 solutions (20 equiv. $\mathbf{M 1}$ and 1 equiv. $\mathbf{G} 3$ in $\mathrm{CD}_{2} \mathrm{Cl}_{2}$; see Supplementary Information) and added 10 equiv. of either CTA1 or CTA2. We then followed the reactions with ${ }^{1} \mathrm{H}$ NMR spectroscopy over time. CTA2 reacts rapidly with POLY-G3 (see Fig. 2a). The POLY-G3 ruthenium carbene signal (18.5 ppm) is completely shifted to a new signal at $19.07 \mathrm{ppm}$ (which corresponds to the G3-benzylidene complex) within $10 \mathrm{~min}$.

In contrast, the identical reaction of CTA1 with POLY-G3 generated the G3-benzylidene ${ }^{1} \mathrm{H}$ NMR signal only very slowly (Fig. 2b; $47 \%$ conversion after $170 \mathrm{~min})$. In separate ${ }^{1} \mathrm{H}$ NMR experiments (see the Supplementary Information), we estimated the kinetic rate constants for the reaction of POLY-G3 with CTA1 $(k=0.001)$ and CTA2 $(k=0.36)$. These data clearly confirm that CTA2 reacts at least 300 times faster with the propagating polymer chain than CTA1. We propose that this vast difference in rate constant could be due to an entropically favoured ring closure in CTA2, due to its bicyclic conformational restriction.

Most likely, the POLY-G3 ruthenium carbene complex and the endocyclic double bond of CTA2 do not undergo a regioselective ring-opening metathesis reaction and therefore give rise to two ring-opened ruthenium alkylidene species (Fig. 3, centre). One pathway (Fig. 3, centre top) yields a ring-opened carbene intermediate that cannot undergo a productive ring-closing metathesis reaction (Fig. 3, centre top, red arrow) as this would form a highly strained norbornene. This carbene intermediate will therefore react back to the starting material and eventually undergo a different ring-opening metathesis reaction yielding another intermediate carbene (Fig. 3, centre bottom). This carbene can undergo a productive ring-closing reaction (Fig. 3, centre bottom, pink arrow) to form a polymer chain with the bicyclic fragment of CTA2 attached to the chain end (POLY-CTA2) via its exocyclic double bond (Fig. 3, bottom right). All in all, the productive reaction pathway shown in Fig. 3 shows substrate selectivity (endocyclic olefin) and pseudo-regioselectivity (as only one ring-opened pathway forms the product).

These findings prompted us to explore CTA2 as a reversible CTA in a catalytic living ROMP. Figure 4 shows the proposed mechanism of a catalytic living ROMP via degenerative chain transfer, using the polymerization of M1 with $\mathbf{G} 3$ as an example. As shown in Fig. 4a, G3 reacts with monomer M1 to form POLY-G3. As the 


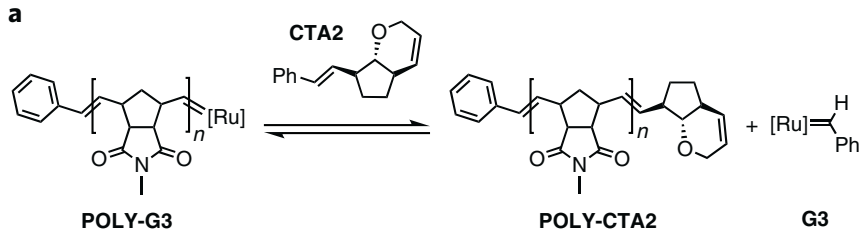

$10 \min (100 \%$ conversion $)$

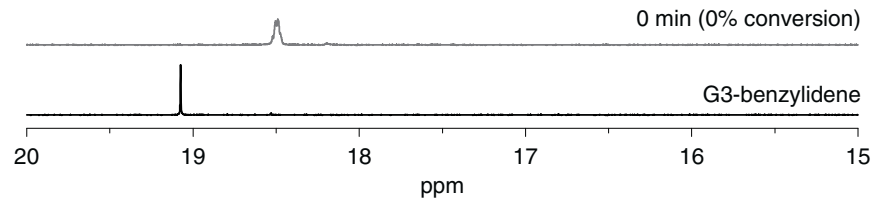

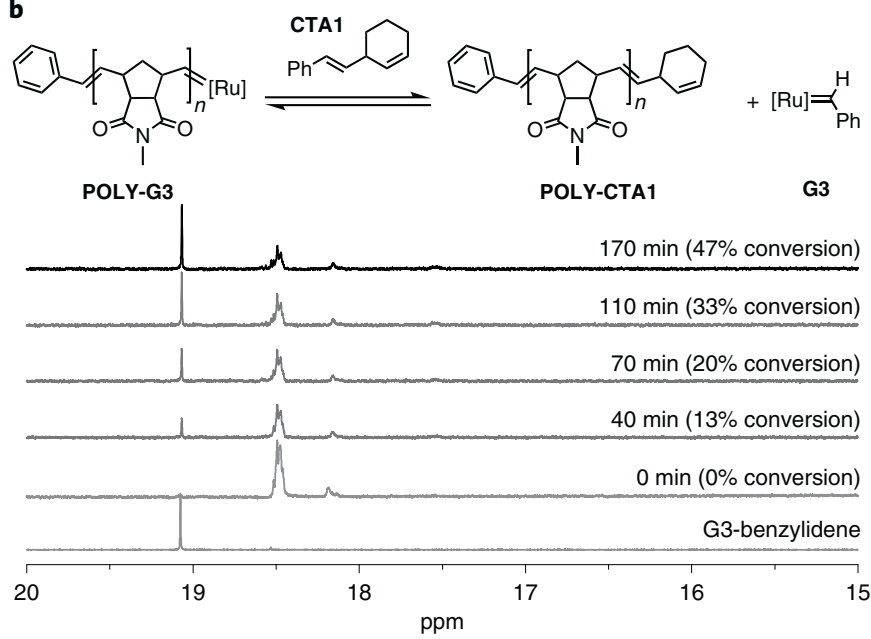

Fig. 2 | ' $\mathrm{H}$ NMR spectra $\left(\mathrm{CD}_{2} \mathrm{Cl}_{2} ; 400 \mathrm{MHz}\right)$ of the reaction of CTA1 or CTA2 with POLY-G3. a, Reaction of the POLY-G3 carbene complex (20 equiv. M1and 1 equiv. $\mathbf{G} 3$ in $\mathrm{CD}_{2} \mathrm{Cl}_{2}$; see Supplementary Information) with 10 equiv. CTA2, yielding the $\mathbf{G} 3$-benzylidene complex (19.07 ppm) within 10 min (100\% conversion). b. Reaction of the POLY-G3 carbene complex with 10 equiv. CTA1, yielding the G3-benzylidene complex (19.07 ppm) within $170 \mathrm{~min}$ (47\% conversion).

monomer is added slowly to the reaction mixture, its concentration is low throughout the polymerization reaction. This dramatically reduces the rate of propagation and hence increases the likelihood of POLY-G3 reacting with the endocyclic double bond of CTA2 in the ring-opening/ring-closing sequence described above. This yields a polymer POLY-CTA2 and regenerates the G3-benzylidene complex. Once all of CTA2 has reacted in this manner, the active polymer POLY-G3 will react with POLY-CTA2 (Fig. 4b).

Reaction of POLY-G3 (Fig. 4b, propagating polymer in red) with POLY-CTA2 (Fig. 4b, in blue) leads to an exchange of end groups (that is, after the reaction, the polymer depicted in blue carries the propagating carbene complex (POLY-G3) and the polymer depicted in red has been reversibly terminated with the bicyclic CTA2 fragment (POLY-CTA2)). Therefore, our polymerization system can be described as follows. Most of the polymer chains can be represented by POLY-CTA2, while only a small number (a catalytic number) of chains can be represented by POLY-G3. Because the propagating ruthenium carbene complexes of POLY-G3 can exchange with any of the chain ends of POLY-CTA2, all polymer chain ends will be randomly and sequentially activated over time. As this process is fast compared with the rate of monomer propagation, it gives the macroscopic impression of all chains growing at the same time.

Inspired by the above concept, the polymerization of M1 was carried out under catalytic living ROMP conditions. Grubbs et al. ${ }^{47}$ had shown that the rate of propagation of ring-strained monomers, such as norbornene derivatives, is significantly faster than the olefin metathesis reaction with acyclic olefins. Therefore, M1 was added slowly via a syringe pump to keep its concentration, and hence the propagation rate $\left(k_{\mathrm{p}}\right)$, low throughout the polymerization process. The slow addition of an M1 (0.45 g, 562 equiv.) solution in dichloromethane (DCM) $\left(0.025 \mathrm{~g} \mathrm{ml}^{-1}\right)$ at a speed of $2 \mathrm{mlh}^{-1}$ to a reaction mixture containing $\mathbf{G 3}(4.52 \mu \mathrm{mol}, 1$ equiv. $)$ and CTA2 (10 equiv.) resulted in the complete consumption of M1 and formation of a polymer P1 with a much lower molecular weight than that calculated from the $[\mathbf{M 1}]:[\mathbf{G} 3]$ ratio. This is an important indicator of a successful degenerative chaintransfer reaction, as in this case the molecular weight should be determined by the [M1]:[CTA2] ratio.

Looking at Fig. 4, one can see that during the initial consumption of CTA2 the intermediate carbene complex (Fig. 4a) is part of a non-degenerative pre-equilibrium. On one side of the pre-equilibrium, POLY-G3 is formed, whereas the G3-benzylidene complex is formed on the other. If an energetic bias towards the POLY-G3 complex existed for this pre-equilibrium, the initial consumption of CTA2 would be retarded, which could potentially lead to a broadening of the final polymer molecular weight distribution.

To investigate the effect of this pre-equilibrium, we synthesized CTA3 (Fig. 1), which carries a cyclohexyl group (instead of the phenyl group in CTA2) attached to the exocyclic double bond but is otherwise identical to CTA2. We believed that at the pre-equilibrium stage (Fig. 4a), a potential energetic bias towards one of the two sides of the equilibrium would be less pronounced for CTA3 than for CTA2. We proposed that during the CTA3 pre-equilibrium, the formation of POLY-G3 versus G3-cyclohexylmethylidene would be energetically similar (in analogy to Fig. 4a). To our surprise, the catalytic living ROMP with G3 and M1 in the presence of CTA3 gave almost the same results as for CTA2 (see Supplementary Table 1), indicating that the non-degenerative pre-equilibrium does not affect the polymerization in these cases.

Next, we varied the $[\mathbf{M 1}]:[$ CTA2] ratio (while keeping the amount of $\mathbf{G 3}(4.52 \mu \mathrm{mol})$ and the [M1]:[G3] ratio (562:1) constant). As expected, different molecular weight polymers (P1-P6) were obtained (analysed by gel permeation chromatography (GPC)). When the molecular weights (analysed by GPC) of the obtained polymers were plotted against the [M1]:[CTA2] ratio, a linear correlation between molecular weight $\left(M_{\mathrm{n}}, \mathrm{GPC}\right)$ and the [M1]:[CTA2] ratio was observed (Fig. 5a, in blue). All polymers obtained showed relatively low molecular weight dispersities.

In addition, polymers P1-P6 were examined by isotopically resolved matrix-assisted laser desorption ionization time-of-flight (MALDI-TOF) mass spectrometry. All obtained masses fitted the proposed polymer structures in which the bicyclic CTA2 fragment is attached to one chain end of the polymer and the phenyl group is attached to the other (see Supplementary Figs. 16-21). When polymer P6 ( $8 \mathrm{kDa} ; Ð=1.33)$ bearing the bicyclic CTA2 fragment at the chain end (acting as POLY-CTA2) was redissolved in DCM along with a new catalytic amount of G3-benzylidene complex and exposed to the slow addition of a second strained monomer (exo-N-hexyl-norbornenecarboximide, M3), a block copolymer P7 $(16 \mathrm{kDa} ; Ð=1.52)$ was formed, as observed by the shift of the GPC trace to a higher molecular weight (see Supplementary Fig. 2). This shows clearly that the reversibly terminated polymer chains can be reactivated at a later stage, which supports the proposed degenerative chain-transfer chain growth mechanism.

Furthermore, the catalytic living ROMP was examined using catalyst G2. Generally, G2 yields ROMP polymers with broad 


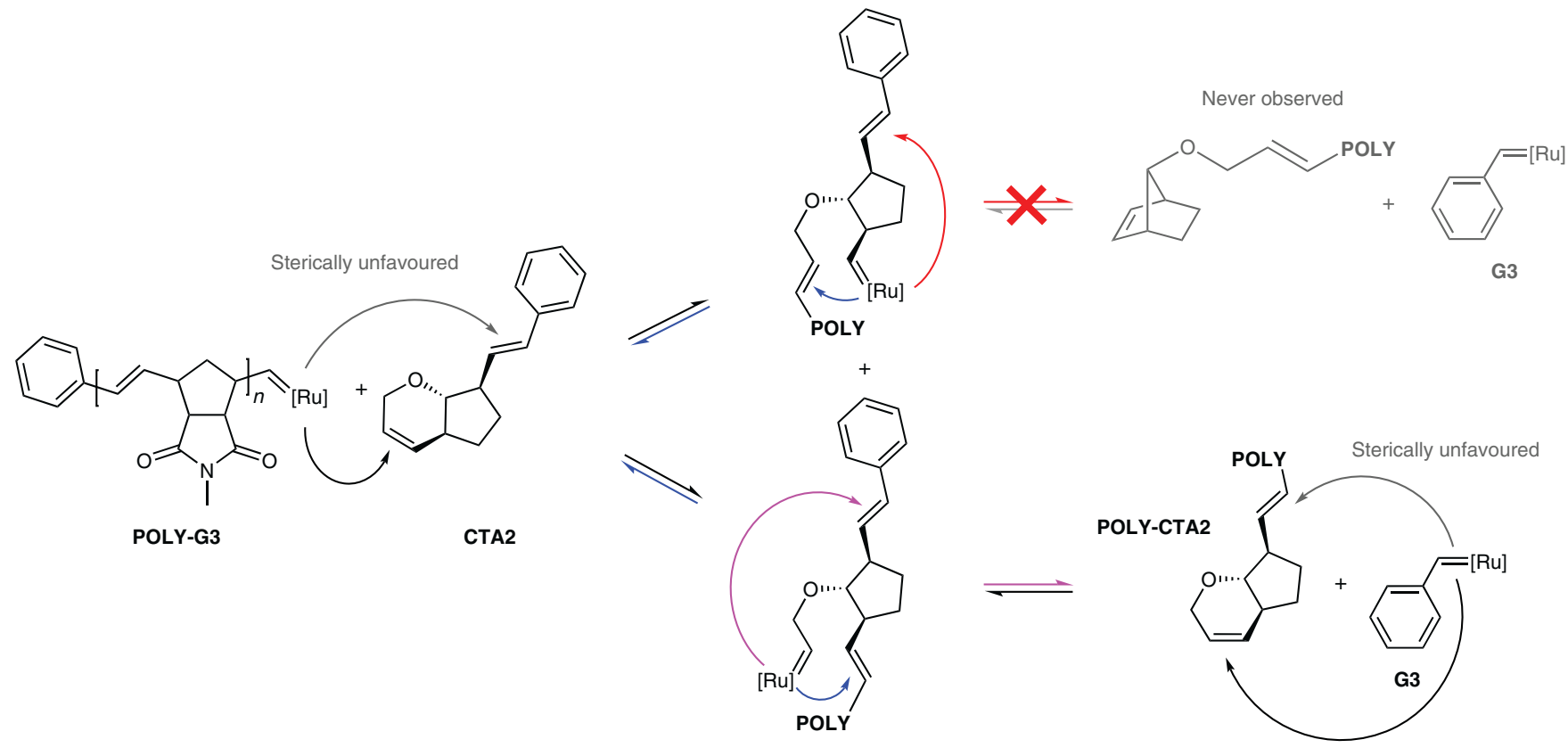

Fig. 3 | Possible reactions of CTA2 with the POLY-G3 ruthenium carbene complex. We propose that the POLY-G3 ruthenium carbene complex (20 equiv. $\mathbf{M 1}$ and 1 equiv. $\mathbf{G} 3$ in $\mathrm{CD}_{2} \mathrm{Cl}_{2}$; see Supplementary Information) can react with the endocyclic double bond of CTA2 and proceed via two reaction pathways. The red reaction pathway (top) is highly disfavoured as it would lead to the formation of a strained norbornene ring. The pink reaction pathway (bottom) leads to the formation of POLY-CTA2 (a polymer chain with the bicyclic fragment of CTA2 as an end group) and the G3-benzylidene complex.

molecular weight dispersities and poorly controlled molecular weights, due to its poor initiation-to-propagation-rate ratio $^{2}$. To our surprise, the catalytic living ROMP using $\mathbf{G 2}$ gave similar results to those observed for the ruthenium complex G3. When varying the $[\mathbf{M 1}]:[\mathbf{C T A 2}]$ ratio, full control over the molecular weight and molecular weight dispersities $(Đ=1.25-1.40)$ was achieved (Fig. 5a, in red). All polymers obtained (P8-P11) were examined by isotopically resolved MALDI-TOF mass spectrometry, and the obtained masses are in agreement with the proposed polymer structures (see Supplementary Figs. 22-25). We believe that under reversible chain-transfer polymerization conditions, any unfavourable ratio between active and inactive catalyst species will only affect the rate of polymerization, and not the final molecular weight dispersity. This method is therefore an example of a controlled living ROMP giving narrow molecular weight dispersities using the second-generation Grubbs' catalyst.

The molecular weight dispersities obtained for polymers P8P11 resemble those of early Grubbs' initiators ${ }^{48}$. However, virtually identical molecular weight dispersites have been reported for cyclopolymerizations of diynes ${ }^{49}$ or the polymerization to yield conjugated polyenynes ${ }^{31}$ with the $\mathbf{G} 3$ initiator in a conventional living ROMP. Other examples include the G3-initiated copolymerization of norbornene derivatives carrying peptides, chromophores and poly(ethylene gylcol) chains in a living fashion, leading to slightly broader molecular weight dispersites ${ }^{30}$ or the polymerization of chromophore-labelled monomers with the G3 complex ${ }^{11}$.

In catalytic living ROMP, all polymer chains carry the reversible CTA at the chain end, which allows chain extension towards block copolymer structures (see above). Additionally, polymers and block copolymers with molecular weight dispersities of $\boxminus \leq 1.40$ can still be sufficiently narrow to be exploited in polymer self-assembly application $s^{50}$. Furthermore, the method presented here is one of very few methods that allow a catalytic ROMP of norbornene derivatives $^{37,47}$. We believe that this polymerization method therefore represents a living polymerization following a reversible deactivation mechanism $^{51}$. In particular, this method will be very beneficial for the synthesis of biomedical polymers due to the reduced amount of toxic ruthenium complexes.

We also examined Grubbs' catalyst $\mathbf{G 1}$ regarding its performance in catalytic living ROMP, but unfortunately polymers with broader molecular weight dispersities and poor molecular weight control were obtained. We believe that this is due to the poor reactivity of G1 towards the low strain endocyclic double bond in CTA2.

To overcome the inconvenience of slow monomer addition via a syringe pump, there are two principle options: either increase the reaction rate of the reversible CTA or decrease the rate of monomer propagation. Previous reports showed that sterically hindered monomers were used to slow their propagation, to achieve alternating architectures with less bulky comonomers ${ }^{52,53}$. To slow the rate of propagation, we chose a bridgehead-substituted norbornene derivative, exo-4-ethyl- $\mathrm{N}$-methyl-7-oxanorbornenecarboximide (M2).

The monomer M2 (307 equiv., $0.025 \mathrm{~g} \mathrm{ml}^{-1}$ in DCM) was added in one portion to a Schlenk flask containing a G2 $(4.71 \mu \mathrm{mol}$, 1 equiv.) and CTA3 solution in DCM $(1 \mathrm{ml})$, and polymerized for $24 \mathrm{~h}$ at room temperature. The experiment was repeated by varying the amount of CTA3 (P12: 20 equiv.; P13: 30 equiv.; P14: 40 equiv.; P15: 50 equiv.) while keeping all other reaction conditions constant. Polymers P12-P15 were obtained in this manner. Figure 5b shows a plot of the number average molecular weight of P12-P15 (GPC in tetrahydrofuran (THF)) versus the [M2]:[CTA3] ratio. Even though the molecular weights obtained are higher than those targeted, the graph shows a linear relationship between $M_{\mathrm{n}}$ and the [M2]:[CTA3] ratio. This indicates that excellent molecular weight control is possible. Furthermore, MALDI-TOF mass spectrometry revealed that all polymer chains show isotopically resolved masses corresponding to the proposed target structure carrying the CTA3 fragment at the chain end.

To show that the degenerative chain-transfer mechanism of catalytic living ROMP is not limited to monomers that do not carry reactive functional groups (M1-M3), we prepared monomers M4-M6. All three of these monomers represent exo-norborneneimides carrying either a triisopropylsilyl-protected primary alcohol (M4), 
<smiles>CC(C)/C=C\c1ccccc1</smiles>

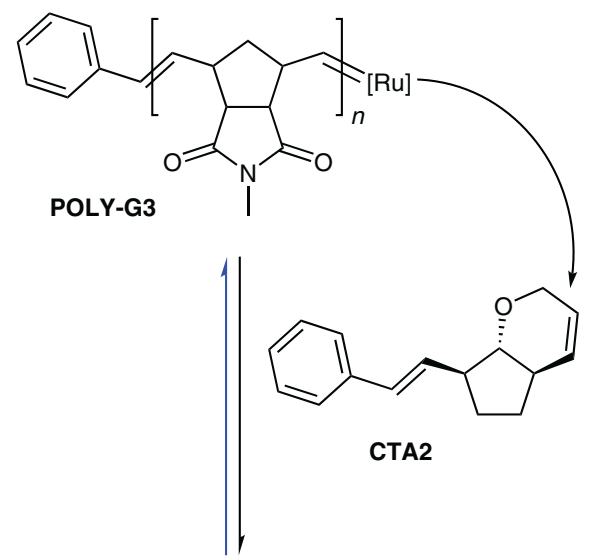

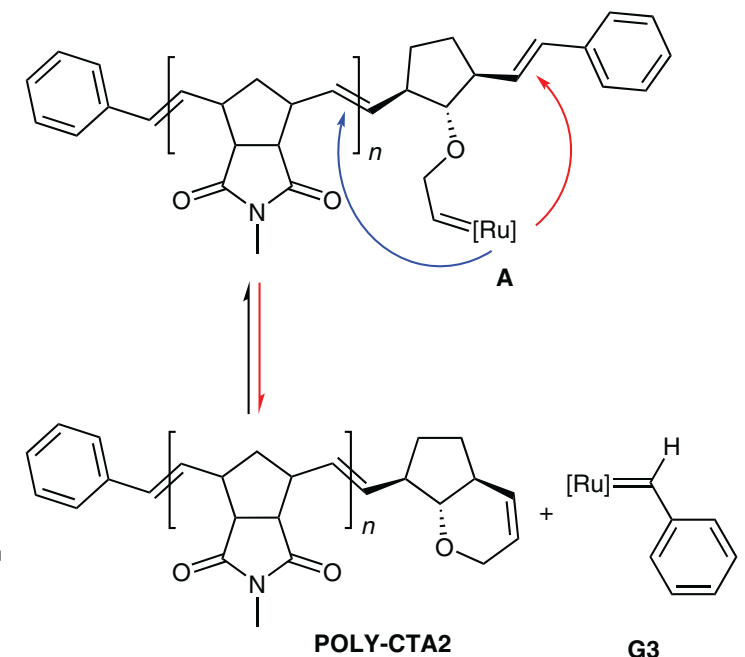<smiles>CN1C(=O)C2C(CC=Cc3ccccc3)CC(CC(C)(C)C)C2C1=O</smiles>

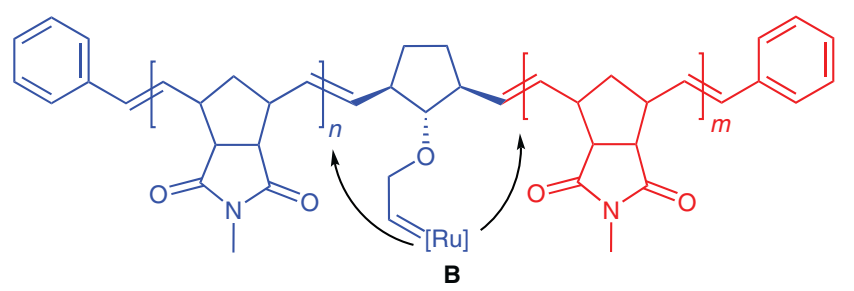

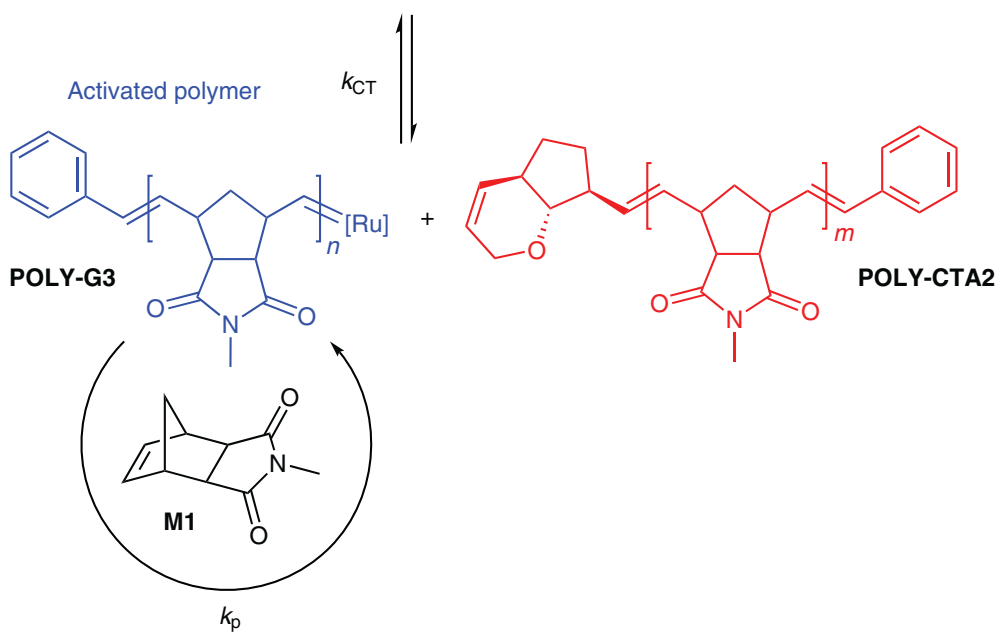

Fig. 4 | Mechanism of catalytic living ROMP, involving degenerative metathesis chain transfer. An example of the mechanism is depicted with catalyst G3 and monomer M1. a, G3 initiates the polymerization of monomer M1, forming POLY-G3. All of CTA2 is consumed by an intermediate ruthenium carbene complex, A, in a non-degenerative pre-equilibrium. The productive reaction (shown in red) yields the G3-benzylidene complex and POLY-CTA2. The back reaction is shown in blue. $\mathbf{b}$, Once all of CTA2 has been converted to POLY-CTA2 (shown in blue), a catalytic number of propagating POLY-G3 chains (shown in red) can react with the end groups of POLY-CTA2 (blue) to form a carbene intermediate, $\mathbf{B}$, via a degenerative equilibrium. The reaction to one side (top) of the equilibrium activates the polymer chain shown in red, whereas the reaction to the other side (bottom) activates the polymer chain shown in blue. $k_{\mathrm{p}}$ is the rate constant of propagation, and $k_{\mathrm{CT}}$ is the rate constant of chain transfer.

a redox-active ferrocene substituent (M5) or the fluorescent coumarin (M6) attached via the imide nitrogen. All three monomers were polymerized under catalytic living ROMP conditions (using G2 and CTA2) and showed excellent molecular weight control according to the [monomer]:[CTA2] ratio (see Supplementary Tables 4-6).

In addition to economical savings, the polymers produced via this method are generally colourless (see Supplementary Fig. 83) due to the low amount of ruthenium complex necessary for their preparation. We therefore believe that this method is an excellent choice for the preparation of highly functional, low molecular weight ROMP polymers, particularly when low amounts of toxic ruthenium residues are required, such as in biomedical applications or whenever metal impurities interfere with electronic or optical properties of the polymers.

\section{Conclusion}

We have developed a new polymerization mechanism for living ring-opening metathesis polymerization requiring only catalytic amounts of Grubbs' second- or third-generation complex. In catalytic living ROMP, we exploited the degenerative metathesis reaction of reversible CTAs. Two reversible CTAs are presented. Polymers with typical molecular weight dispersities $<1.4$ and molecular weights determined by the monomer-to-CTA ratio were obtained. This method allows the synthesis of living ring-opening 

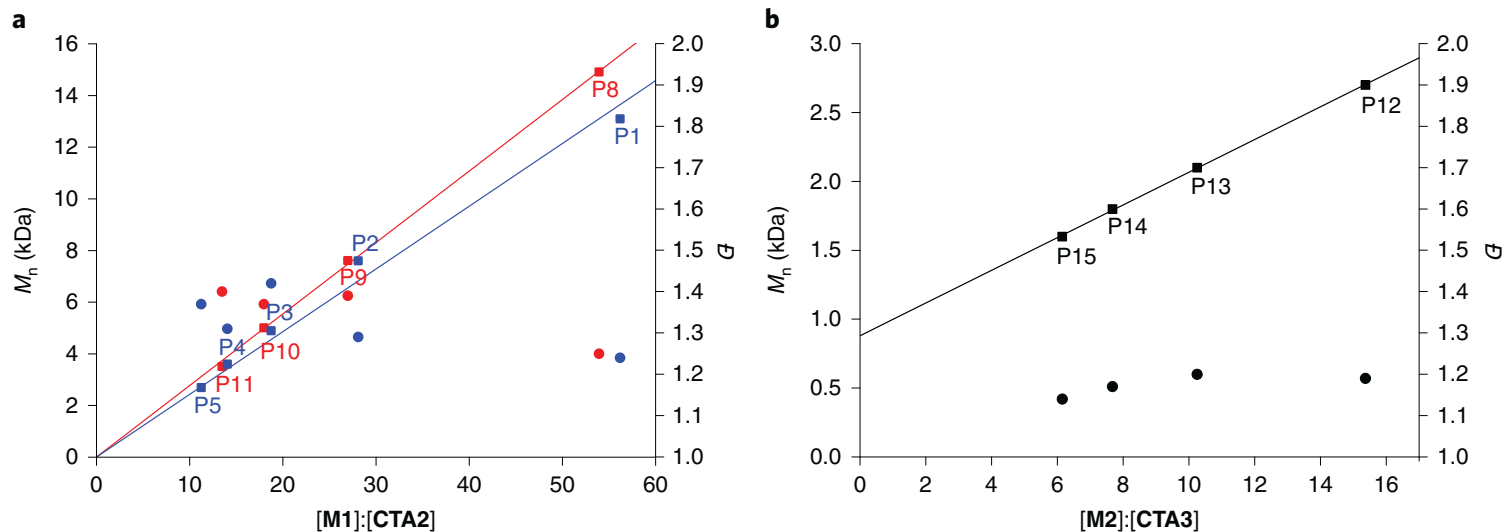

Fig. 5 | Plots of the polymer number average molecular weight versus the monomer-to-CTA ratio. a, Polymerizations with M1, CTA2 and catalyst G3 (blue) or G2 (red). GPC data were measured in chloroform. Low polydispersity and a linear correlation between the molecular weight of the polymers and the [M1]:[CTA2] ratio are indicative of a living polymerization under reversible chain-transfer conditions. The polymers shown in blue were synthesized by keeping the amounts of $\mathbf{G 3}$ (4.52 $\mu$ mol, 1 equiv.) and $\mathbf{M} 1$ (562 equiv.) constant, while changing the equivalents of CTA2 (P1, 10 equiv.; P2, 20 equiv.; P3, 30 equiv.; P4, 40 equiv.; P5, 50 equiv.). The polymers shown in red were synthesized by keeping the amounts of $\mathbf{G 2}(4.71 \mu \mathrm{mol}, 1$ equiv.) and $\mathbf{M 1}$ (539 equiv.) constant, while changing the equivalents of CTA2 (P8, 10 equiv.; P9, 20 equiv.; P10, 30 equiv.; P11, 40 equiv.). b, Polymerizations with M2, CTA3 and G2. GPC data were measured in THF. A linear correlation was observed between the molecular weight of the polymers and different [M2]:[CTA3] ratios - a requirement for a successful degenerative chain-transfer polymerization. The polymers shown were synthesized by keeping the amounts of $\mathbf{G 2}$ ( $4.71 \mu \mathrm{mol}, 1$ equiv.) and $\mathbf{M 2}$ (307 equiv.) constant, while changing the equivalents of CTA3 (P12, 20 equiv.; P13, 30 equiv.; P14, 40 equiv.; P15, 50 equiv.). Squares refer to the molecular weight axis (left), whereas circles refer to the molecular weight dispersity axis (right). Each data point corresponds to one measurement. See Supplementary Tables 1-3 for detailed polymerization conditions.

metathesis polymers and also block copolymers using substoichiometric amounts of ruthenium complex. The living polymerization with narrow molecular weight dispersities using Grubbs' secondgeneration catalyst is particularly important, as this complex does not give low-polydispersity polymers in the absence of the degenerative chain-transfer mechanism described here. To reduce the monomer propagation rate with respect to the rate of reversible chain transfer, the monomer is typically added slowly to the polymerization mixture. Using a sterically hindered monomer with slow propagation characteristics, we were able to synthesize living polymers under catalytic conditions without the need for slow monomer addition. Furthermore, highly functional monomers carrying ferrocene, coumarin or a triisopropylsilyl-protected primary alcohol in the side chain were also successfully polymerized under living catalytic ROMP conditions. All polymers were fully characterized by GPC (in chloroform or THF) and isotopically resolved MALDITOF mass spectrometry, confirming the proposed degenerative chain-transfer mechanism.

This method provides a more economical way to synthesize low molecular weight ROMP polymers by reducing the loading of expensive ruthenium complexes for their synthesis. Due to the low amount of residual ruthenium complex, the polymers obtained by this method are less coloured and less toxic than those prepared by conventional living ROMP. This economical and environmentally friendly method could therefore lead to new applications in materials science, biology or medicine.

\section{Methods}

A typical procedure for the catalytic living ring-opening metathesis polymerization with either $\mathbf{G} 2$ or $\mathbf{G} 3$ catalyst is described as follows. Schlenk flasks containing monomer M1 $(0.45 \mathrm{~g}, 2.54 \mathrm{mmol}, 561.8$ equiv.) and a mixture of CTA2 (10-50 equiv.; see Supplementary Tables 1 and 2) and either G2 (4 mg, $4.71 \mu \mathrm{mol}, 1$ equiv.) or $\mathbf{G} 3$ catalyst ( $4 \mathrm{mg}, 4.52 \mu \mathrm{mol}, 1$ equiv.) were closed, evacuated and backfilled with argon three times. A solution of the deoxygenated monomer M1 in dry, degassed DCM (4.5-18.0 ml; see Supplementary Tables 1 and 2) was then added to a mixture of G3 and CTA2 in dry, degassed DCM $(1 \mathrm{ml})$ at room temperature using a syringe pump, which maintained the flow rate at $2 \mathrm{ml} \mathrm{h}^{-1}$. Immediately after the addition of the monomer, active metathesis species were quenched with ethyl vinyl ether (222-231 equiv.). The crude product was concentrated in vacuo, precipitated into cold methanol and dried under high vacuum.

\section{Data availability}

All data generated or analysed during this study are included within the article and its Supplementary Information files.

Received: 16 April 2018; Accepted: 27 February 2019;

Published online: 8 April 2019

\section{References}

1. Sutthasupa, S., Shiotsuki, M. \& Sanda, F. Recent advances in ring-opening metathesis polymerization, and application to synthesis of functional materials. Polym. J. 42, 905-915 (2010).

2. Choi, T.-L. \& Grubbs, R. H. Controlled living ring-opening-metathesis polymerization by a fast-initiating ruthenium catalyst. Angew. Chem. Int. Ed. 42, 1743-1746 (2003).

3. Chen, Y., Abdellatif, M. M. \& Nomura, K. Olefin metathesis polymerization: some recent developments in the precise polymerizations for synthesis of advanced materials (by ROMP, ADMET). Tetrahedron 74, 619-643 (2018).

4. Schrock, R. R. in Handbook of Metathesis 2nd edn, Vol. 1 (eds Grubbs, R. H. \& Wenzel, A. G.) 1-27 (Wiley, 2015).

5. Buchmeiser, M. R. Molybdenum imido, tungsten imido and tungsten oxo alkylidene $N$-heterocyclic carbene olefin metathesis catalysts. Chem. Eur. J. 24, 14295-14301 (2018)

6. Ogba, O. M., Warner, N. C., O'Leary, D. J. \& Grubbs, R. H. Recent advances in ruthenium-based olefin metathesis. Chem. Soc. Rev. 47, 4510-4544 (2018).

7. Demel, S., Schoefberger, W., Slugovc, C. \& Stelzer, F. Benchmarking of ruthenium initiators for the ROMP of a norbornenedicarboxylic acid ester. J. Mol. Catal. A 200, 11-19 (2003).

8. Trzaskowski, B. \& Grela, K. Structural and mechanistic basis of the fast metathesis initiation by a six-coordinated ruthenium catalyst. Organometallics 32, 3625-3630 (2013).

9. Gu, H. et al. Tetrablock metallopolymer electrochromes. Angew. Chem. Int. Ed. 57, 2204-2208 (2018).

10. Liaw, D.-J., Wang, K.-L., Lee, K.-R. \& Lai, J.-Y. Ring-opening metathesis polymerization of new norbornene-based monomers containing various chromophores. J. Polym. Sci. A 45, 3022-3031 (2007).

11. Riga, E. K. et al. Fluorescent ROMP monomers and copolymers for biomedical applications. Macromol. Chem. Phys. 218, 1700273 (2017).

12. Nguyen, H. V.-T. et al. Scalable synthesis of multivalent macromonomers for ROMP. ACS Macro Lett. 7, 472-476 (2018). 
13. Schaefer, M., Hanik, N. \& Kilbinger, A. F. M. ROMP copolymers for orthogonal click functionalizations. Macromolecules 45, 6807-6818 (2012).

14. Yang, S. Y. \& Weck, M. Modular covalent multifunctionalization of copolymers. ACS Macro Lett. 41, 346-351 (2008).

15. Kumar, D. R., Lidster, B. J., Adams, R. W. \& Turner, M. L. Understanding the microstructure of poly( $p$-phenylenevinylene)s prepared by ring-opening metathesis polymerization using ${ }^{13} \mathrm{C}$-labeled paracyclophanediene monomers. Macromolecules 51, 4572-4577 (2018).

16. Su, J. K. et al. Synthesis and mechanochemical activation of ladderenenorbornene block copolymers. J. Am. Chem. Soc. 140, 12388-12391 (2018).

17. Schaefer, M. et al. The role of mass and length in the sonochemistry of polymers. Macromolecules 49, 1630-1636 (2016).

18. Foster, J. C., Varlas, S., Blackman, L. D., Arkinstall, L. A. \& O’Reilly, R. K. Ring-opening metathesis polymerization in aqueous media using a macroinitiator approach. Angew. Chem. Int. Ed. 57, 10672-10676 (2018).

19. Lee, H.-K. et al. Superior cascade ring-opening/ring-closing metathesis polymerization and multiple olefin metathesis polymerization: enhancing the driving force for successful polymerization of challenging monomers. J. Am. Chem. Soc. 140, 10536-10545 (2018).

20. Park, H. \& Choi, T.-L. Fast tandem ring-opening/ring-closing metathesis polymerization from a monomer containing cyclohexene and terminal alkyne. J. Am. Chem. Soc. 134, 7270-7273 (2012).

21. Park, H., Lee, J.-K. \& Choi, T.-L. Tandem ring-opening/ring-closing metathesis polymerization: relationship between monomer structure and reactivity. J. Am. Chem. Soc. 135, 10769-10775 (2013).

22. Park, H., Kang, E.-H., Müller, L. \& Choi, T.-L. Versatile tandem ringopening/ring-closing metathesis polymerization: strategies for successful polymerization of challenging monomers and their mechanistic studies. J. Am. Chem. Soc. 138, 2244-2251 (2016).

23. Elling, B. R. \& Xia, Y. Efficient and facile end group control of living ring-opening metathesis polymers via single addition of functional cyclopropenes. ACS Macro Lett. 7, 656-661 (2018).

24. Pal, S., Lucarini, F., Ruggi, A. \& Kilbinger, A. F. M. Functional metathesis catalyst through ring closing enyne metathesis: one pot protocol for living heterotelechelic polymers. J. Am. Chem. Soc. 140, 3181-3185 (2018).

25. Zhang, T., Fu, L. \& Gutekunst, W. R. Practical synthesis of functional metathesis initiators using enynes. Macromolecules 41, 6497-6504 (2018).

26. Nagarkar, A. \& Kilbinger, A. F. M. End functional ROMP polymers via degradation of a ruthenium Fischer type carbene. Chem. Sci. 5, 4687-4692 (2014)

27. Hilf, S., Grubbs, R. H. \& Kilbinger, A. F. M. End capping ring-opening olefin metathesis polymerization polymers with vinyl lactones. J. Am. Chem. Soc. 130, 11040-11048 (2008).

28. Smith, D., Pentzer, E. B. \& Nguyen, S. T. Bioactive and therapeutic ROMP polymers. Polym. Rev. 47, 419-459 (2007).

29. Sarapas, J. M., Backlund, C. M., deRonde, B. M., Minter, L. M. \& Tew, G. N. ROMP- and RAFT-based guanidinium-containing polymers as scaffolds for protein mimic synthesis. Chem. Eur. J. 23, 6858-6863 (2017).

30. Xie, N. et al. A simple, modular synthesis of bifunctional peptidepolynorbornenes for apoptosis induction and fluorescence imaging of cancer cells. Polym. Chem. 9, 77-86 (2018).

31. Kang, C., Park, H., Lee, J. K. \& Choi, T. L. Cascade polymerization via controlled tandem olefin metathesis/metallotropic 1,3-shift reactions for the synthesis of fully conjugated polyenynes. J. Am. Chem. Soc. 139, 11309-11312 (2017)

32. Kang, C., Kang, E. H. \& Choi, T. L. Successful cyclopolymerization of 1,6-heptadiynes using first-generation Grubbs catalyst twenty years after its invention: revealing a comprehensive picture of cyclopolymerization using Grubbs catalysts. Macromolecules 50, 3153-3163 (2017).

33. Yang, S., Shin, S., Choi, I., Lee, J. \& Choi, T.-L. Direct formation of large-area 2D nanosheets from fluorescent semiconducting homopolymer with orthorhombic crystalline orientation. J. Am. Chem. Soc. 139, 3082-3088 (2017).

34. Kovacic, S., Kren, H., Krajnc, P., Koller, S. \& Slugovc, C. The use of an emulsion templated microcellular poly(dicyclopentadiene-co-norbornene) membrane as a separator in lithium-ion batteries. Macromol. Rapid Commun. 34, 581-587 (2013)

35. Zha, Y. P., Disabb-Miller, M. L., Johnson, Z. D., Hickner, M. A. \& Tew, G. N. Metal-cation-based anion exchange membranes. J. Am. Chem. Soc. 134, 4493-4496 (2012).

36. Bielawski, C. W., Benitez, D., Morita, T. \& Grubbs, R. H. Synthesis of end-functionalized poly(norbornene)s via ring-opening metathesis polymerization. Macromolecules 34, 8610-8618 (2001).
37. Liu, P., Yasir, M., Ruggi, A. \& Kilbinger, A. F. M. Heterotelechelic polymers by ring-opening metathesis and regioselective chain transfer. Angew. Chem. Int. Ed. 57, 914-917 (2018).

38. Chiefari, J. et al. Living free-radical polymerization by reversible additionfragmentation chain transfer: the RAFT process. Macromolecules 31, 5559-5562 (1998).

39. Zhang, Y. H., Keaton, R. J. \& Sita, L. R. Degenerative transfer living Ziegler-Natta polymerization: application to the synthesis of monomodal stereoblock polyolefins of narrow polydispersity and tunable block length. J. Am. Chem. Soc. 125, 9062-9069 (2003).

40. Kempe, R. How to polymerize ethylene in a highly controlled fashion? Chem. Eur. J. 13, 2764-2773 (2007).

41. Valente, A., Mortreux, A., Visseaux, M. \& Zinck, P. Coordinative chain transfer polymerization. Chem. Rev. 113, 3836-3857 (2013).

42. Ajellal, N. et al. Metal-catalyzed immortal ring-opening polymerization of lactones, lactides and cyclic carbonates. Dalton Trans. 39, 8363-8376 (2010)

43. Uchiyama, M., Satoh, K. \& Kamigaito, M. Cationic RAFT polymerization using ppm concentrations of organic acid. Angew. Chem. Int. Ed. 54, 1924-1928 (2015).

44. Nagarkar, A. A. \& Kilbinger, A. F. M. Catalytic living ring-opening metathesis polymerization. Nat. Chem. 7, 718-723 (2015).

45. Nagarkar, A. A. \& Kilbinger, A. F. M. Retraction note: catalytic living ring-opening metathesis polymerization. Nat. Chem. 10, 573 (2018).

46. Nagarkar, A. A., Yasir, M., Crochet, A., Fromm, K. M. \& Kilbinger, A. F. M. Tandem ring-opening-ring-closing metathesis for functional metathesis catalysts. Angew. Chem. Int. Ed. 55, 12343-12346 (2016).

47. Matson, J. B., Virgil, S. C. \& Grubbs, R. H. Pulsed-addition ring-opening metathesis polymerization: catalyst-economical syntheses of homopolymers and block copolymers. J. Am. Chem. Soc. 131, 3355-3362 (2009).

48. Kanaoka, S. \& Grubbs, R. H. Synthesis of block copolymers of siliconcontaining norbornene derivatives via living ring-opening metathesis polymerization catalyzed by a ruthenium carbene complex. Macromolecules 28, 4707-4713 (1995).

49. Song, J.-A. \& Choi, T.-L. Seven-membered ring-forming cyclopolymerization of 1,8-nonadiyne derivatives using Grubbs catalysts: rational design of monomers and insights into the mechanism for olefin metathesis polymerizations. Macromolecules 50, 2724-2735 (2017).

50. Doncom, K. E., Blackman, L. D., Wright, D. B., Gibson, M. I. \& O’Reilly, R. K. Dispersity effects in polymer self-assemblies: a matter of hierarchical control. Chem. Soc. Rev. 46, 4119-4134 (2017).

51. Jenkins, A. D., Jones, R. G. \& Moad, G. Terminology for reversibledeactivation radical polymerization previously called 'controlled' radical or 'living' radical polymerization (IUPAC recommendations 2010). Pure Appl. Chem. 82, 483-491 (2010).

52. Daeffler, C. S. \& Grubbs, R. H. Catalyst-dependent routes to ring-opening metathesis alternating copolymers of substituted oxanorbornenes and cyclooctene. Macromolecules 46, 3288-3292 (2013).

53. Elling, B. R. \& Xia, Y. Living alternating ring-opening metathesis polymerization based on single monomer additions. J. Am. Chem. Soc. 137, 9922-9926 (2015)

\section{Acknowledgements}

A.F.M.K., M.Y. and P.L. thank the Swiss National Science Foundation for funding. I.K.T. thanks the National of Competence in Research 'Bio-inspired Nanomaterials' for a postdoctoral fellowship.

\section{Author contributions}

M.Y., P.L. and A.F.M.K. designed the experiments. M.Y. and P.L. performed the experiments. M.Y., I.K.T. and A.F.M.K. wrote the main manuscript text. All authors reviewed the manuscript.

\section{Competing interests}

The authors declare no competing interests.

\section{Additional information}

Supplementary information is available for this paper

Correspondence and requests for materials should be addressed to A.F.M.K. 